\title{
A note on explicit expressions for moments of order statistics
}

\author{
Fredy Castellares ${ }^{1}$, Artur J. Lemonte ${ }^{2}$, Marcos A.C. Santos ${ }^{3}$ \\ ${ }^{1}$ Universidade Federal de Minas Gerais, Belo Horizonte/MG, Brazil, fwcc29@gmail.com \\ 2 Universidade Federal do Rio Grande do Norte, Natal/RN, Brazil, arturlemonte@gmail.com \\ ${ }^{3}$ Universidade Federal de Minas Gerais, Belo Horizonte/MG, Brazil, msantos@est.ufmg.br
}

Received: August 30, 2021. Accepted: December 20, 2021.

\begin{abstract}
Closed-form expressions for moments of order statistics from the normal, log-normal, gamma and beta distributions were provided in the statistics literature. In particular, the explicit expressions involve the Lauricella function of type A, and the generalized Kampé de Fériet function. We note that the expressions provided by the author do not appear correct, which implies that the expressions cannot be recommended to users. An alternative closed-form expression for moments of order statistics is then provided. We also consider numerical studies to show that the formulas we provide deliver satisfactory results.
\end{abstract}

Keywords: Kampé de Fériet function, Lauricella function

MSC: 60E05

\section{Moments of order statistics}

Let $X_{1}, X_{2}, \ldots, X_{n}$ be a random sample of size $n$ from some random variable $X$ with probability density function (PDF) given by $f$, and cumulative distribution function (CDF) given by $F$. Let $X_{1: n}<X_{2: n}<\cdots<X_{n: n}$ denote the corresponding order statistics. The PDF of $X_{r: n}$ is given by (see, for example, David and Nagaraja, 2003)

$$
f_{r: n}(x)=\frac{n !}{(r-1) !(n-r) !} \sum_{m=0}^{n-r}(-1)^{m}\left(\begin{array}{c}
n-r \\
m
\end{array}\right) F^{m+r-1}(x) f(x), \quad x \in \mathbb{R},
$$

where $r=1,2, \ldots, n$. The moments of order statistics are

$$
\mathbb{E}\left(X_{r: n}^{k}\right)=\frac{n !}{(r-1) !(n-r) !} \sum_{m=0}^{n-r}(-1)^{m}\left(\begin{array}{c}
n-r \\
m
\end{array}\right) \int_{-\infty}^{\infty} x^{k} F^{m+r-1}(x) f(x) d x
$$


where $k=1,2, \ldots$ and $r=1,2, \ldots, n$; that is,

$$
\mathbb{E}\left(X_{r: n}^{k}\right)=\frac{n !}{(r-1) !(n-r) !} \sum_{m=0}^{n-r}(-1)^{m}\left(\begin{array}{c}
n-r \\
m
\end{array}\right) I(k, m-r+1),
$$

where

$$
I(k, s)=\int_{-\infty}^{\infty} x^{k} F^{s}(x) f(x) d x, \quad k, s \in \mathbb{N} .
$$

In general, the above integral has no closed-form analytical solution and, hence, the use of special functions like Lauricella function of type A and (generalized) Kampé de Fériet function to express $I(k, s)$ in closed-form are welcome. However, one has to use these special functions with some caution, mainly with respect to the convergence radius of these functions, otherwise, they will be divergent and so the use of them becomes inviable.

The Lauricella function of type A Exton (1978) is defined as

$$
F_{A}^{(n)}\left(a ; b_{1}, \ldots, b_{n}, c_{1}, \ldots, c_{n} ; x_{1}, \ldots, x_{n}\right)=\sum_{i_{1}, \ldots, i_{n}=0}^{\infty} \frac{(a)_{i_{1}+\cdots+i_{n}}\left(b_{1}\right)_{i_{1}} \cdots\left(b_{n}\right)_{i_{n}}}{\left(c_{1}\right)_{i_{1}} \cdots\left(c_{n}\right)_{i_{n}} i_{1} ! \cdots i_{n} !} x_{1}^{i_{1}} \cdots x_{n}^{i_{n}},
$$

where $(q)_{n}$ is the (rising) Pochhammer symbol, i.e. $(q)_{0}=1$, and $(q)_{n}=q(q+1) \cdots(q+n-1)$ for $n \geq 1$. We have that the convergence radius of the Lauricella function of type $\mathrm{A}$ is

$$
\left|x_{1}\right|+\cdots+\left|x_{n}\right|<1 .
$$

Therefore, if the above condition is not satisfied, the Lauricella function of type A will divergent for any values of $a, b_{1}, \ldots, b_{n}$ and $c_{1}, \ldots, c_{n}$. For example, if the $n$ arguments of the Lauricella function in expression (2) are all equal to -1 , we have $\left|x_{1}\right|+\cdots+\left|x_{n}\right|=n \geq 1$ and, hence, we have that

$$
F_{A}^{(n)}\left(a ; b_{1}, \ldots, b_{n}, c_{1}, \ldots, c_{n} ;-1,-1, \ldots,-1\right)=+\infty .
$$

\section{Results from Nadarajah (2008)}

In the following, some closed-form expressions for $\mathbb{E}\left(X_{r: n}^{k}\right)$ derived by Nadarajah (2008) are presented.

\subsection{Moments of normal order statistics}

Nadarajah (2008, eq. (12)) arrived at the following closed-form expression for $\mathbb{E}\left(X_{r: n}^{k}\right)$ :

$$
\begin{aligned}
\mathbb{E}\left(X_{r: n}^{k}\right)= & \frac{n !}{(r-1) !(n-r) !} \sum_{l=0}^{n-r}\left(\begin{array}{c}
n-r \\
l
\end{array}\right)\left(-\frac{1}{2}\right)^{l} \sum_{\substack{p=0 \\
p+k \text { even }}}^{r+l-1}\left(\begin{array}{c}
r+l-1 \\
p
\end{array}\right) \\
& \times(\pi)^{(1-p) / 2} 2^{p} \Gamma\left(\frac{k+p+1}{2}\right) F_{A}^{(p)}\left(\frac{k+p+1}{2} ; \frac{1}{2}, \ldots, \frac{1}{2} ; \frac{3}{2}, \ldots, \frac{3}{2} ;-1, \ldots,-1\right),
\end{aligned}
$$

where $\Gamma(\cdot)$ is the complete gamma function. Note that the $(p)$ arguments of the Lauricella function in expression (3) are all equal to -1 . From (3), it is evident that

$$
F_{A}^{(p)}\left(\frac{k+p+1}{2} ; \frac{1}{2}, \ldots, \frac{1}{2} ; \frac{3}{2}, \ldots, \frac{3}{2} ;-1, \ldots,-1\right)=+\infty,
$$

which invalidates the formula (3) for $\mathbb{E}\left(X_{r: n}^{k}\right)$ obtained by Nadarajah (2008). 


\subsection{Moments of lognormal order statistics}

Nadarajah (2008, eq. (21)) arrived at the following closed-form expression for $\mathbb{E}\left(X_{r: n}^{k}\right)$ :

$$
\begin{aligned}
\mathbb{E}\left(X_{r: n}^{k}\right)= & \frac{n ! 2^{1-r}}{(r-1) !(n-r) !} \exp \left(\frac{k^{2}}{2}\right) \sum_{l=0}^{n-r}\left(-\frac{1}{2}\right)^{l}\left(\begin{array}{c}
n-r \\
l
\end{array}\right) \sum_{p=0}^{r+l-1}\left(\begin{array}{c}
r+l-1 \\
p
\end{array}\right)\left(-\frac{2}{\sqrt{\pi}}\right)^{p} \\
& \times \mathbb{E}\left[\left(\frac{N \sqrt{2}-k}{2 \sqrt{2}}\right)^{p} F_{A}^{(p)}\left(\frac{1}{2}, \ldots, \frac{1}{2} ; \frac{3}{2}, \ldots, \frac{3}{2} ;-\frac{(N \sqrt{2}-k)^{2}}{8}, \ldots,-\frac{(N \sqrt{2}-k)^{2}}{8}\right)\right],
\end{aligned}
$$

where $N$ is a standard normal random variable. The above expression for $\mathbb{E}\left(X_{r: n}^{k}\right)$ does not converge. Note that the Lauricella function depends on random arguments, given by the quantity

$$
\frac{(N \sqrt{2}-k)^{2}}{8}
$$

In this case, it is not possible to ensure that

$$
\left|\frac{(N \sqrt{2}-k)^{2}}{8}\right|+\cdots+\left|\frac{(N \sqrt{2}-k)^{2}}{8}\right|<1,
$$

which is the convergence radius of the Lauricella function.

\subsection{Moments of gamma order statistics}

Nadarajah (2008, eq. (26)) arrived at the following closed-form expression for $\mathbb{E}\left(X_{r: n}^{k}\right)$ :

$$
\begin{aligned}
\mathbb{E}\left(X_{r: n}^{k}\right)= & \frac{n !}{(r-1) !(n-r) !} \sum_{l=0}^{n-r}\left(\begin{array}{c}
n-r \\
l
\end{array}\right) \Gamma(\alpha)^{-r-l} \alpha^{1-r-l} \Gamma(k+\alpha(r+l)) \\
& \times F_{A}^{(r+l-1)}(k+\alpha(r+l) ; \alpha, \ldots, \alpha, \alpha+1, \ldots, \alpha+1 ;-1, \ldots,-1) .
\end{aligned}
$$

Note that the $(r+l-1)$ arguments of the Lauricella function of type A in expression (4) are all equal to -1 , which implies that we can have $\left|x_{1}\right|+\cdots+\left|x_{r+l-1}\right|=r+l-1>1$ for $r=1,2, \ldots, n$ and $l=0,1, \ldots, n-r$. In this case, the Lauricella function of type $\mathrm{A}$ is also divergent, which invalidates expression (4) for $\mathbb{E}\left(X_{r: n}^{k}\right)$ derived by Nadarajah (2008).

\subsection{Moments of beta order statistics}

Nadarajah (2008, eq. (31)) arrived at the following closed-form expression for $\mathbb{E}\left(X_{r: n}^{k}\right)$ :

$$
\begin{aligned}
& \mathbb{E}\left(X_{r: n}^{k}\right)=\frac{n !}{(r-1) !(n-r) !} \sum_{l=0}^{n-r}(-1)^{l}\left(\begin{array}{c}
n-r \\
l
\end{array}\right) a^{1-r-l} B(a, b)^{-r-l} B(b, k+a(r+l)) \\
& \times F_{1: 1}^{1: 2}\left(\begin{array}{c}
(k+a(r+l)):(1-b, a) ; \cdots:(1-b, a) ; 1,1, \ldots, 1), \\
(b+k+a(r+l)):(1+a) ; \cdots:(1+a) ;
\end{array},\right.
\end{aligned}
$$

where $B(\cdot, \cdot)$ is the beta function, and $F_{1: 1}^{1: 2}(\cdot)$ is the Kampé de Fériet function, which is a generalization of the generalized hypergeometric series, introduced by Joseph Kampé de Fériet; see, for 
example, Exton (1978, eq. (1.4.2)). The $F_{1: 1}^{1: 2}(\cdot)$ function is of the form

$$
\begin{aligned}
F_{C: D}^{A: B}:= & F_{1: 1}^{1: 2}\left(\begin{array}{rl}
(a): \overbrace{\left(b_{1}, b_{2}\right) ; \cdots:\left(b_{1}, b_{2}\right)}^{n} & n \\
(c): \underbrace{(d) ; \cdots:(d)}_{n} ; & \left.x_{1}, x_{2}, \ldots, x_{n}\right)
\end{array}\right)= \\
& \sum_{i_{1}=0}^{\infty} \cdots \sum_{i_{n}=0}^{\infty} \frac{(a)_{i_{1}+\cdots+i_{n}} \prod_{j=1}^{n}\left(b_{1}\right)_{i_{j}} \prod_{j=1}^{n}\left(b_{2}\right)_{i_{j}}}{(c)_{i_{1}+\cdots+i_{n}} \prod_{j=1}^{n}(d)_{i_{j}}} \frac{x_{1}^{i_{1}} \cdots x_{n}^{i_{n}}}{i_{1} ! \cdots i_{n} !} .
\end{aligned}
$$

The convergence radius of the Kampé de Fériet function is as follows (see, for example, Exton, 1978, p. 26): if $A+B=C+D+1$ and $A=C$, the Kampé de Fériet function converges at the region

$$
\left|x_{1}\right|<1, \ldots,\left|x_{n}\right|<1 .
$$

However, we have that all arguments of the Kampé de Fériet function in expression (5) are all equal to 1 , which implies that

$$
F_{1: 1}^{1: 2}\left(\begin{array}{c}
(k+a(r+l)):(1-b, a) ; \cdots:(1-b, a) ; 1,1, \cdots, 1)=+\infty . \\
(b+k+a(r+l)):(1+a) ; \cdots:(1+a) ;
\end{array}\right)
$$

Therefore, the expression (5) for $\mathbb{E}\left(X_{r: n}^{k}\right)$ derived by Nadarajah (2008) is not valid.

\section{An alternative expression}

Here, let $X_{1}, X_{2}, \ldots, X_{n}$ be a random sample of size $n$ identically distributed sampled from a random variable $X$, with CDF given by $F$. Let $X_{1: n}<X_{2: n}<\cdots<X_{n: n}$ denote the corresponding order statistics. Let $S$ be the suport of $X$. Assume that the CDF $F$ can be evaluated using a convergent power series expansion (with convergence radius $\rho>0$ ) of the form

$$
F(x)=\sum_{j=0}^{\infty} b_{j} x^{j}, \quad x \in S \quad \text { and } \quad S \subseteq(-\rho, \rho),
$$

where $b_{j}(j=0,1, \ldots)$ are coefficients that can depend on the model parameters. For example, for random variables with support $S=(0, \infty)$ (as the gamma distribution, for example), we have that $\rho=\infty$. It is worth stressing that the result derived in this section cannot be applied without the above assumptions. A simple example where the result derived in this section cannot be applied is the Cauchy distribution. The Cauchy CDF is

$$
F(x)=\frac{1}{\pi} \arctan \left(\frac{x-\mu}{\theta}\right)+\frac{1}{2}, \quad x \in(-\infty, \infty),
$$

where $-\infty<\mu<\infty$ and $\theta>0$. The above CDF does not admit a convergent power series expansion, and so our result cannot be applied.

Now, consider an equation for a power series raised to a positive integer $s$ (see, for example Gradshteyn and Ryzhik, 2007)

$$
\left(\sum_{j=0}^{\infty} b_{j} x^{j}\right)^{s}=\sum_{j=0}^{\infty} b_{j}^{(s)} x^{j}, \quad x \in S,
$$


where

$$
b_{j}^{(s)}=\sum_{k=0}^{j} b_{k}^{(s-1)} b_{j-k}, \quad b_{j}^{(1)}:=b_{j}, \quad b_{j}^{(0)}= \begin{cases}1, & j=0, \\ 0, & j>0 .\end{cases}
$$

We can express the PDF of $X_{r: n}$ in (1) as

$$
f_{r: n}(x)=\frac{n !}{(r-1) !(n-r) !} \sum_{m=0}^{n-r}(-1)^{m}\left(\begin{array}{c}
n-r \\
m
\end{array}\right) \sum_{j=0}^{\infty} b_{j}^{(m+r-1)} x^{j} f(x), \quad x \in S .
$$

The moments of order statistics become

$$
\mathbb{E}\left(X_{r: n}^{k}\right)=\frac{n !}{(r-1) !(n-r) !} \sum_{m=0}^{n-r}(-1)^{m}\left(\begin{array}{c}
n-r \\
m
\end{array}\right) I(k, m-r+1),
$$

where

$$
I(k, s)=\sum_{j=0}^{\infty} b_{j}^{(s)} \int_{S} x^{k+j} f(x) d x
$$

Note that

$$
\int_{S} x^{k+j} f(x) d x=\mathbb{E}\left(X^{k+j}\right)
$$

and so it follows that

$$
\mathbb{E}\left(X_{r: n}^{k}\right)=\frac{n !}{(r-1) !(n-r) !} \sum_{m=0}^{n-r}(-1)^{m}\left(\begin{array}{c}
n-r \\
m
\end{array}\right) \sum_{j=0}^{\infty} b_{j}^{(m+r-1)} \mathbb{E}\left(X^{k+j}\right) .
$$

For example, from expression (7), the moments of the maximum value of the sample $\left(X_{n: n}\right)$ take the form

$$
\mathbb{E}\left(X_{n: n}^{k}\right)=n \sum_{j=0}^{\infty} b_{j}^{(n-1)} \mathbb{E}\left(X^{j+k}\right), \quad k=1,2, \ldots
$$

Also, for $n=1$, the above expression reduces exactly to the moments of the random variable $X$, which is given by $\mathbb{E}\left(X^{k}\right)$, for $k=1,2, \ldots$.

\section{Examples}

\subsection{Gamma distribution}

Here, we assume that $X_{1}, X_{2}, \ldots, X_{n}$ are $n$ identically distributed gamma random variables, and $X_{1: n}<X_{2: n}<\cdots<X_{n: n}$ are the corresponding order statistics. The lower incomplete gamma function can be evaluated using the convergent power series expansion

$$
\gamma(\alpha, x)=\exp (-x) x^{\alpha} \Gamma(\alpha) \sum_{j=0}^{\infty} \frac{x^{j}}{\Gamma(\alpha+j+1)}, \quad x>0,
$$

and, hence, the gamma CDF can be expressed as

$$
F(x)=\frac{\gamma(\alpha, x)}{\Gamma(\alpha)}=\exp (-x) x^{\alpha} \sum_{j=0}^{\infty} \frac{x^{j}}{\Gamma(\alpha+j+1)}, \quad x>0 .
$$


Let $b_{j}=[\Gamma(\alpha+j+1)]^{-1}$, for $j=0,1, \ldots$. We have that

$$
F^{m+r-1}(x)=\exp [-(m+r-1) x] x^{(m+r-1) \alpha}\left(\sum_{j=0}^{\infty} b_{j} x^{j}\right)^{m+r-1} .
$$

Note that $b_{0}=[\Gamma(\alpha+1)]^{-1} \neq 0$, and so (8) is convergent for all $x>0$ and convergence radius $\rho=\infty$. Hence, we can express the PDF of $X_{r: n}$ in (1) as

$$
f_{r: n}(x)=\frac{n !}{(r-1) !(n-r) !} \sum_{m=0}^{n-r}(-1)^{m}\left(\begin{array}{c}
n-r \\
m
\end{array}\right) x^{\alpha(m+r-1)} \mathrm{e}^{-(m+r-1) x} \sum_{j=0}^{\infty} b_{j}^{(m+r-1)} x^{j} f(x),
$$

where $x>0$. The moments of gamma order statistics are

$$
\mathbb{E}\left(X_{r: n}^{k}\right)=\frac{n !}{(r-1) !(n-r) !} \sum_{m=0}^{n-r}(-1)^{m}\left(\begin{array}{c}
n-r \\
m
\end{array}\right) \sum_{j=0}^{\infty} \frac{b_{j}^{(m+r-1)}}{\Gamma(\alpha)} \int_{0}^{\infty} x^{k+\alpha(m+r)+j-1} \mathrm{e}^{-(m+r) x} d x .
$$

After some algebra, we arrive at the following closed-form expression:

$$
\mathbb{E}\left(X_{r: n}^{k}\right)=\frac{n !}{(r-1) !(n-r) !} \sum_{m=0}^{n-r} \frac{(-1)^{m}}{\Gamma(\alpha)}\left(\begin{array}{c}
n-r \\
m
\end{array}\right) \sum_{j=0}^{\infty} b_{j}^{(m+r-1)} \frac{\Gamma(\alpha(m+r)+j+k)}{(m+r)^{\alpha(m+r)+j+k}} .
$$

For example, the moments of the maximum value of the sample take the form

$$
\mathbb{E}\left(X_{n: n}^{k}\right)=\frac{1}{\Gamma(\alpha)} \sum_{j=0}^{\infty} b_{j}^{(n-1)} \frac{\Gamma(\alpha n+j+k)}{n^{n \alpha+k+j-1}}, \quad k=1,2, \ldots
$$

Also, for $n=1$, the above expression reduces exactly to the moments of a single gamma random variable, which is given by $\Gamma(\alpha+k) / \Gamma(\alpha)$, for $k=1,2, \ldots$.

\subsection{Beta distribution}

Here, we assume that $X_{1}, X_{2}, \ldots, X_{n}$ are $n$ identically distributed beta random variables, and $X_{1: n}<$ $X_{2: n}<\cdots<X_{n: n}$ are the corresponding order statistics. The beta CDF can be expressed as

$$
F(x)=\frac{x^{a}}{B(a, b)} \sum_{j=0}^{\infty} \frac{(1-b)_{j}}{(a+j) j !} x^{j}, \quad 0<x<1,
$$

where $B(a, b)=\int_{0}^{1} u^{a-1}(1-u)^{b-1} d u$ is the beta function, and $a>0$ and $b>0$. Define $(\alpha)_{n}=$ $(\alpha)(\alpha+1) \cdots(\alpha+n-1)$ for $n \in \mathbb{N}, \alpha \in \mathbb{R}$, and $(\alpha)_{0}=1$. Let $b_{j}=(1-b)_{j} /[(a+j) j !]$, for $j=0,1, \ldots$ We have that

$$
F^{m+r-1}(x)=\frac{x^{a(m+r-1)}}{B(a, b)^{m+r-1}}\left(\sum_{j=0}^{\infty} b_{j} x^{j}\right)^{m+r-1} .
$$

Note that $b_{0}=a^{-1} \neq 0$, and so (10) is convergent for all $0<x<1$ and convergence radius $\rho=1$. Hence, we can express the PDF of $X_{r: n}$ in (1) as

$$
f_{r: n}(x)=\frac{n !}{(r-1) !(n-r) !} \sum_{m=0}^{n-r}(-1)^{m}\left(\begin{array}{c}
n-r \\
m
\end{array}\right) \frac{x^{\alpha(m+r-1)}}{B(a, b)^{m+r-1}} \sum_{j=0}^{\infty} b_{j}^{(m+r-1)} x^{j} f(x),
$$


where $0<x<1$.

The moments of beta order statistics are

$$
\mathbb{E}\left(X_{r: n}^{k}\right)=\frac{n !}{(r-1) !(n-r) !} \sum_{m=0}^{n-r}(-1)^{m}\left(\begin{array}{c}
n-r \\
m
\end{array}\right) \sum_{j=0}^{\infty} \frac{b_{j}^{(m+r-1)}}{B(a, b)^{m+r}} \int_{0}^{1} x^{k+a(m+r)+j-1}(1-x)^{b-1} d x .
$$

After some algebra, we arrive at the following closed-form expression:

$$
\mathbb{E}\left(X_{r: n}^{k}\right)=\frac{n !}{(r-1) !(n-r) !} \sum_{m=0}^{n-r}(-1)^{m}\left(\begin{array}{c}
n-r \\
m
\end{array}\right) \sum_{j=0}^{\infty} b_{j}^{(m+r-1)} \frac{B(k+a(m+r)+j, b)}{B(a, b)^{m+r}} .
$$

For example, the moments of the maximum value of the sample take the form

$$
\mathbb{E}\left(X_{n: n}^{k}\right)=n \sum_{j=0}^{\infty} b_{j}^{(n-1)} \frac{B(k+a n+j, b)}{B(a, b)^{n}}, \quad k=1,2, \ldots
$$

Also, for $n=1$, the above expression reduces exactly to the moments of a single beta random variable, which is given by $B(a+k, b) / B(a, b)$, for $k=1,2, \ldots$.

\section{Numerical results}

In this section, we provide numerical values for some moments of gamma order statistics. The scripts were written in the $\mathrm{R}$ program (R Core Team, 2020). We use the proposed closed-form expansion (9) to obtain numerical approximations for $\mathbb{E}\left(X_{r: n}^{k}\right)$. Table 1 lists the values of $b_{j}=[\Gamma(\alpha+j+1)]^{-1}$ and $b_{j}^{(s)}$ (obtained using the recurrence relation (6)) for $j=0,1, \ldots, 6$ and $s=0,1, \ldots, 6$ of a gamma distribution with $\alpha=1$. Table 2 lists numerical values of $\mathbb{E}\left(X_{r: 6}^{k}\right)$ for $k=1$ and 2 obtained by computational implementation of expansion (9) for a gamma distribution with $\alpha=6.401$, which is the same value used for numerical calculations in Nadarajah (2008). Table 3 presents numerical values of $\mathbb{E}\left(X_{r: 10}\right)$ for $r=1,2, \ldots, 10$, sample size $n=10$ and $\alpha=6.401$. In Tables 2 and 3, we also compare the numerical moments obtained from (9) with those calculated from $R$ code by using direct numerical integration (last columns in Tables 2 and 3). Note that the numerical values are near, mainly when $j_{\max }=100$.

Table 1: $b_{j}^{(s)}$ coefficients for a gamma distribution with $\alpha=1$.

\begin{tabular}{ccccccccc}
\hline$j$ & $b_{j}$ & $b_{j}^{(0)}$ & $b_{j}^{(1)}$ & $b_{j}^{(2)}$ & $b_{j}^{(3)}$ & $b_{j}^{(4)}$ & $b_{j}^{(5)}$ & $b_{j}^{(6)}$ \\
\hline 0 & 1.000000 & 1 & 1.000000 & 1.000000 & 1.000000 & 1.000000 & 1.000000 & 1.000000 \\
1 & 0.500000 & 0 & 0.500000 & 1.000000 & 1.500000 & 2.000000 & 2.500000 & 3.000000 \\
2 & 0.166667 & 0 & 0.166667 & 0.583333 & 1.250000 & 2.166667 & 3.333333 & 4.750000 \\
3 & 0.041667 & 0 & 0.041667 & 0.250000 & 0.750000 & 1.666667 & 3.125000 & 5.250000 \\
4 & 0.008333 & 0 & 0.008333 & 0.086111 & 0.358333 & 1.012500 & 2.298611 & 4.529167 \\
5 & 0.001389 & 0 & 0.001389 & 0.025000 & 0.143750 & 0.513889 & 1.406250 & 3.237500 \\
6 & 0.000198 & 0 & 0.000198 & 0.006300 & 0.050017 & 0.225562 & 0.741733 & 1.989616 \\
\hline
\end{tabular}


Table 2: $\mathbb{E}\left(X_{r: 6}^{k}\right)$ obtained by the proposed expansion with $j_{\max }$ terms for a gamma distribution with $\alpha=6.401$. The last column shows the corresponding values by numerical integration.

\begin{tabular}{ccccc}
\hline$k$ & $r$ & $j_{\max }=60$ & $j_{\max }=100$ & $\mathbb{E}\left(X_{r: 6}^{k}\right)$ \\
\hline 1 & 1 & 3.6038 & 3.5882 & 3.5878 \\
1 & 2 & 4.5781 & 4.7153 & 4.7173 \\
1 & 3 & 6.0764 & 5.6749 & 5.6708 \\
1 & 4 & 6.1215 & 6.6589 & 6.6632 \\
1 & 5 & 8.2274 & 7.8864 & 7.8841 \\
1 & 6 & 9.8048 & 9.8884 & 9.8889 \\
2 & 1 & 14.1859 & 14.0150 & 14.0062 \\
2 & 2 & 21.3902 & 23.3735 & 23.4198 \\
2 & 3 & 40.0213 & 33.5971 & 33.4992 \\
2 & 4 & 36.8939 & 45.9763 & 46.0795 \\
2 & 5 & 70.5991 & 64.6280 & 64.5737 \\
2 & 6 & 101.2353 & 102.7357 & 102.7471 \\
\hline
\end{tabular}

Table 3: $\mathbb{E}\left(X_{r: 10}\right)$ obtained by the proposed expansion with $j_{\max }$ terms for a gamma distribution with $\alpha=6.401$. The last column shows the corresponding values by numerical integration.

\begin{tabular}{cccc}
\hline$r$ & $j_{\max }=60$ & $j_{\max }=100$ & $\mathbb{E}\left(X_{r: 10}\right)$ \\
\hline 1 & 3.0374 & 3.1384 & 3.1301 \\
2 & 4.6470 & 3.9578 & 3.9992 \\
3 & 4.2703 & 4.6399 & 4.6560 \\
4 & 2.7310 & 5.6396 & 5.2467 \\
5 & 7.7786 & 5.1628 & 5.8255 \\
6 & 11.7461 & 6.2690 & 6.4285 \\
7 & 1.7441 & 8.6948 & 7.0954 \\
8 & 3.5427 & 5.9839 & 7.8888 \\
9 & 16.1067 & 9.9258 & 8.9495 \\
10 & 8.4161 & 10.6081 & 10.8002 \\
\hline
\end{tabular}

Table 4: $\mathbb{E}\left(X_{r: 6}^{k}\right)$ for a beta distribution with parameters $a=2, b=1.2$, obtained by the correspondent expansion with $j_{\max }$ terms. The last column shows the values by numerical integration.

\begin{tabular}{ccccc}
\hline$k$ & $r$ & $j_{\max }=60$ & $j_{\max }=100$ & $\mathbb{E}\left(X_{r: 6}^{k}\right)$ \\
\hline 1 & 1 & 0.306093 & 0.306093 & 0.306093 \\
1 & 2 & 0.465444 & 0.465444 & 0.465444 \\
1 & 3 & 0.589541 & 0.589551 & 0.589552 \\
1 & 4 & 0.697362 & 0.697439 & 0.697461 \\
1 & 5 & 0.796690 & 0.796966 & 0.797120 \\
1 & 6 & 0.894870 & 0.894507 & 0.894329 \\
2 & 1 & 0.116362 & 0.116362 & 0.116362 \\
2 & 2 & 0.238599 & 0.238599 & 0.238599 \\
2 & 3 & 0.366921 & 0.366930 & 0.366931 \\
2 & 4 & 0.502333 & 0.502409 & 0.502430 \\
2 & 5 & 0.646928 & 0.647201 & 0.647354 \\
2 & 6 & 0.807429 & 0.807071 & 0.806894 \\
\hline
\end{tabular}


In a similar way, we use the proposed expansion for the moments of beta order statistics to obtain some numerical values using the coefficients $b_{j}$ for $j=0,1, \ldots, j_{\max }$ according to (11). Table 4 lists the values of $\mathbb{E}\left(X_{r: n}^{k}\right)$ for some values of $k, n$ and $r$ for a beta distribution with parameters $a=2$ and $b=1.2$. We compare the numerical results from (11) with those calculated by using direct numerical integration. In this particular example, we can verify a faster convergence rate for the expression (11).

\section{Acknowledgments}

The authors would like to thank the Editor and an anonymous reviewer for their insightful comments and suggestions. Fredy Castellares gratefully acknowledges the financial support from Fundação de Amparo à Pesquisa do Estado de Minas Gerais (FAPEMIG). Artur Lemonte gratefully acknowledges the financial support of the Conselho Nacional de Desenvolvimento Científico e Tecnológivo (CNPq, grant 304776/2019-0).

\section{References}

David, Herbert A and Haikady N Nagaraja (2003). Order statistics (3rd Edition ed.). John Wiley \& Sons.

Exton, Horwood (1978). Handbook of hypergeometric integrals: theory, applications, tables, computer programs. Halsted Press, New York.

Gradshteyn, Izrail Solomonovich and Iosif Moiseevich Ryzhik (2007). Table of integrals, series, and products. Academic press, New York.

Nadarajah, Saralees (2008). Explicit expressions for moments of order statistics. Statistics \& Probability Letters 78(2), 196-205.

R Core Team (2020). R: A language and environment for statistical computing. 